\title{
Infecções comunitárias do trato urinário: prevalência e susceptibilidade aos antimicrobianos na cidade de Florianópolis
}

\author{
Community urinary tract infections: prevalence and antimicrobial susceptibility in Florianópolis \\ Infecciones comunitarias del tracto urinario: prevalencia y susceptibilidad antimicrobiana en

\section{Florianópolis}

Débora Monteiro dos Santos Alves. Santa Casa de Misericórdia de Porto Alegre. Porto Alegre, RS, Brasil.

E-mail: deh_alves@hotmail.com (Autora correspondente)

Marcos Krahe Edelweiss. Prefeitura Municipal de Florianópolis. Florianópolis, SC, Brasil. E-mail: marcosedelweiss@gmail.com Lúcio José Botelho. Universidade Federal de Santa Catarina (UFSC). Florianópolis, SC, Brasil. E-mail: lucio.botelho@ufsc.br

\section{Resumo}

Objetivo: Determinar a frequência de isolamento dos uropatógenos e avaliar o perfil de sensibilidade antimicrobiana in vitro das bactérias isoladas em uroculturas de pacientes ambulatoriais atendidos em Florianópolis no ano de 2014, correlacionando tais dados com sexo e idade do paciente. Métodos: Estudo observacional, descritivo do tipo transversal, que incluiu uroculturas positivas de pacientes ambulatoriais realizadas em um único laboratório seguindo a mesma metodologia. Realizaram-se análises de estatísticas descritivas e testes de associação entre as variáveis classificatórias: sexo, idade, agente etiológico e sensibilidade aos antimicrobianos. Resultados: Foram registradas 1035 uroculturas positivas, das quais $89,66 \%$ de pacientes femininas. Microrganismos Gram-negativos foram os patógenos mais isolados, com destaque para a Escherichia coli $(77,10 \%)$, principal agente causal. Proteus mirabilis foi significativamente mais prevalente no sexo masculino e o principal microrganismo isolado nas uroculturas dos meninos de 1 a 5 anos. A E. coli foi significativamente mais prevalente no sexo feminino e apresentou o perfil mais amplo de resistência. Evidenciaram-se diferenças estatisticamente significativas nas resistências às fluoroquinolonas entre os gêneros, com maiores prevalências de resistência em homens e entre as faixas etárias, com maior resistência entre idosos. Conclusões: Idade e sexo são variáveis determinantes na frequência de isolamento dos uropatógenos e na prevalência da susceptibilidade antimicrobiana; e os microrganismos mais prevalentes já não respondem satisfatoriamente a parte dos antimicrobianos amplamente utilizados. É fundamental que o médico considere tais informações no momento da decisão terapêutica e preze pelo uso racional dos antimicrobianos, sobretudo aqueles de largo espectro como as fluoroquinolonas.
Palavras-chave: Infecções Urinárias Infecções por Enterobacteriaceae Antibiograma Resistência Microbiana a Medicamentos
Como citar: Alves DMS, Edelweiss MK, Botelho LJ. Infecções comunitárias do trato urinário: prevalência e susceptibilidade aos antimicrobianos na cidade de Florianópolis. Rev Bras Med Fam Comunidade. 2016;11(38):1-12. http://dx.doi.org/10.5712/rbmfc11(38)1187
Fonte de financiamento: declaram não haver. Parecer CEP: 1.053.771 (FESSC), aprovado em 07/05/2015.

Conflito de interesses: declaram não haver.

Procedência e revisão por pares: revisado por pares. Recebido em: 04/08/2015. Aprovado em: 26/05/2016. 


\begin{abstract}
Objectives: Determine the isolation frequency of uropathogens and evaluate the antimicrobial susceptibility profile in vitro of the bacteria isolated from urine cultures of outpatients treated in Florianópolis in 2014, correlating these data with gender and age of the patient. Methods: This is an observational, descriptive cross-sectional study which included positive urine cultures of outpatients performed in a single laboratory using the same methodology. Descriptive statistic analyses and tests of association between categorical variables: gender, age, etiologic agent and antimicrobial susceptibility were performed. Results: Positive urine cultures were registered in 1035 patients, of which $89.66 \%$ are female. Gram-negative pathogens were more isolated, especially E. coli $(77.10 \%)$, the main causal agent. P. mirabilis was significantly more prevalent in males and the main microorganism isolated in urine cultures of 1-5 years-old children. E. coli was significantly more prevalent in females and presented the wider profile of resistance. This study showed statistically significant differences in resistance to fluoroquinolones between genders, with higher resistance prevalence in men and between age groups, with higher resistance among the elderly. Conclusions: Age and gender are determinant variables in the isolation frequency of uropathogens and in the prevalence of antimicrobial susceptibility; and the most prevalent microorganisms have not satisfactorily respond to the part of antimicrobials used. It is essential that the physician use such information at the time of therapeutic decision and respect the rational use of antimicrobials, especially those of broad spectrum as fluoroquinolones.
\end{abstract}

\section{Resumen}

Objetivos: Determinar la frecuencia de aislamiento de patógenos urinarios y evaluar el perfil de susceptibilidad antimicrobiana in vitro de las bacterias aisladas de urocultivos de pacientes ambulatorios tratados en Florianópolis, en 2014, correlacionando estos datos con el género y la edad del paciente. Métodos: Estudio observacional, descriptivo, transversal que incluyó urocultivos positivos de pacientes externos realizado en un mismo laboratorio utilizando igual metodología. Fueron realizados análisis estadísticos descriptivos y pruebas de asociación entre las variables usadas para agrupar los pacientes por: género, edad, agente etiológico y susceptibilidad antimicrobiana. Resultados: Se registraron 1035 cultivos de orina positivos, de los cuales $89,66 \%$ de pacientes de sexo femenino. Los patógenos más frecuentes fueron Gram-negativos, especialmente E. coli $(77,10 \%)$, en destaque como principal agente causal. Proteus mirabilis fue significativamente más frecuente en el sexo masculino y el principal microorganismo aislado en urocultivos de niños de 1-5 años. E. coli fue significativamente más frecuente en mujeres y presentó el perfil de resistencia antimicrobiana más amplio. Observamos diferencias estadísticamente significativas cuanto a resistencia a las fluoroquinolonas entre los patógenos de muestras de pacientes masculinos y femeninos, con una prevalencia mayor de resistencia en los cultivos de hombres, y entre los grupos etarios, con mayor prevalencia de resistencia en ancianos. Conclusiones: Edad y género son variables clave para el diagnóstico, por determinar la frecuencia de aislamiento de los diversos patógenos urinarios y la prevalencia de la sensibilidad a los antimicrobianos; y los microorganismos más prevalentes no responden satisfactoriamente a algunos grupos de antimicrobianos. Es esencial que el médico haga uso de tales informaciones en el momento de la decisión terapéutica y evalúe el uso racional de los antimicrobianos, en especial los de amplio espectro como las fluoroquinolonas.

\section{Keywords:}

Urinary Tract Infections

Enterobacteriaceae infections Microbial Sensitivity Tests Drug Resistance, Microbial
Palabras clave:

Infecciones Urinarias

Infecciones por

Enterobacteriaceae

Pruebas de Sensibilidad

Microbiana

Farmacorresistencia

Microbiana

\section{Introdução}

A infecção do trato urinário (ITU), definida como a multiplicação microbiana com invasão tecidual de qualquer segmento do aparelho urinário, desde a uretra até os rins, ${ }^{1}$ constitui uma das principais causas de consulta médica e de prescrição de antimicrobianos, o que acarreta grandes impactos socioeconômicos individuais e sociais. ${ }^{2}$ Em Florianópolis, SC, um levantamento da demanda nas unidades básicas de saúde apontou essa afecção como o 14ํㅡำ motivo de consulta no município, ficando atrás, entre as causas infecciosas, apenas da infecção aguda do aparelho respiratório superior e da gastroenterite. ${ }^{3}$ Casuísticas europeias consideram a ITU como a segunda causa mais comum de indicação de tratamento antimicrobiano empírico na atenção primária ${ }^{4}$ e, em um estudo realizado no sul do Brasil, essa condição foi responsável por 13,3\% das prescrições de antibióticos, número menor apenas que o das infecções das vias aéreas superiores e amigdalites. ${ }^{5}$

A urocultura com antibiograma é o único exame capaz de avaliar a sensibilidade bacteriana aos antimicrobianos e o crescimento de 100.000 unidades formadoras de colônia por mililitro (UFC/mL) representa ITU na grande maioria das vezes. ${ }^{6}$ A alta incidência, a sintomatologia desconfortável e a 
demora do resultado dos exames fazem, porém, com que a terapia seja usualmente iniciada antes que a cultura e o antibiograma sejam conhecidos. ${ }^{2,6,7}$ Além disso, diretrizes estabelecem que infecções baixas não complicadas em mulheres devem ser tratadas empiricamente. ${ }^{8}$

A decisão racional por tratamento empírico requer o conhecimento de quais são os principais agentes etiológicos envolvidos e qual é o perfil de resistência aos antibióticos. ${ }^{9}$ Esse monitoramento periódico é de fundamental importância, visto que os uropatógenos e o padrão de sensibilidade aos antimicrobianos podem variar com o tempo e em cada região geográfica por estarem associados a pressões seletivas locais. ${ }^{10}$ Além disso, tem-se verificado em diversos países uma importante redução da sensibilidade aos antimicrobianos mais empregados, ${ }^{9-11}$ o que torna a escolha terapêutica empírica inadequada um dos erros mais cometidos na prática médica, ${ }^{6}$ resultando em maior morbimortalidade e custos ao sistema de saúde..$^{11,12}$

No Brasil há uma escassez desses dados e a maioria dos trabalhos publicados referem-se a infecções nosocomiais, o que torna as decisões terapêuticas a nível ambulatorial dependentes das diretrizes internacionais, as quais se mostraram inadequadas para países em desenvolvimento. ${ }^{13,14}$ Preocupada com esse cenário, a Organização Mundial de Saúde preconiza a elaboração de estratégias nacionais ou municipais e a criação de laboratórios de referência. ${ }^{5}$ Baseado nessas recomendações, o presente estudo objetiva determinar a frequência de isolamento dos uropatógenos e avaliar o perfil de sensibilidade antimicrobiana in vitro das bactérias isoladas de uroculturas de pacientes ambulatoriais atendidos em Florianópolis no ano de 2014, correlacionando tais dados com o sexo e a idade do paciente, a fim de identificar opções terapêuticas empíricas adequadas.

\section{Métodos}

Trata-se de um estudo observacional, descritivo do tipo transversal, que incluiu uroculturas positivas com antibiograma realizadas, por pedidos oriundos de pacientes ambulatoriais, em um único laboratório seguindo a mesma metodologia, no município de Florianópolis, entre janeiro a dezembro de 2014.

O laboratório de análises clínicas onde o presente estudo foi realizado faz exames ambulatoriais tanto para o Sistema Único de Saúde (SUS) quanto para o setor privado da saúde, não sendo o único que presta serviços ao SUS na cidade. Apresenta uma estrutura com mais de 30 unidades de atendimento (incluindo clínicas e hospitais). Em 2014, foram realizadas nessa instituição 4603 uroculturas, por pedidos oriundos de pacientes ambulatoriais, sendo utilizados protocolos para urocultura e antibiograma conforme recomendações do Infectious Diseases Society of America Guidelines (IDSA), ${ }^{8}$ American Society of Microbiology (ASM), ${ }^{15}$ Clinical and Laboratory Standards Institute (CLSI) ${ }^{16}$ e Nota técnica da anvisa № 01/2013. ${ }^{17}$

O instrumento de pesquisa usado foi uma planilha de recolhimento de dados, sendo as variáveis classificatórias: sexo, idade, agente etiológico e sensibilidade aos antimicrobianos. Não se obteve acesso ao prontuário dos pacientes, sendo assim, variáveis relevantes como: manifestações clínicas (motivo da solicitação do exame), gravidade do quadro, comorbidades associadas, história de infecção prévia e exposição recente a antibióticos não foram analisadas.

Foram incluídos na pesquisa todos os resultados das amostras de urina coletadas de jato urinário médio e/ou final ou através de cateterismo vesical, sendo consideradas positivas as uroculturas que apresentaram crescimento bacteriano superior a $100.000 \mathrm{UFC} / \mathrm{ml}$. 
As ITUs verdadeiramente polimicrobianas são infrequentes, embora não raras. A presença de duas ou mais bactérias na cultura lança dúvidas em relação à confiabilidade do exame e gera a suspeita de uma possível contaminação da amostra. A confirmação do diagnóstico deve ser realizada com amostras seriadas de urina, observando-se a presença dos mesmos patógenos nas diversas amostras. ${ }^{18}$ Como não se obteve acesso a amostras seriadas, optou-se por excluir as uroculturas mistas.

As solicitações de urocultura se restringiram a pedidos oriundos da atenção primária, sendo excluídos do estudo os exames realizados nas unidades hospitalares do laboratório. As culturas com crescimento fúngico também constituíram fator de exclusão e, para efeitos de análise, a susceptibilidade intermediária foi incluída como resistência.

Os dados foram armazenados no programa Microsoft Excel e analisados utilizando o software Epi Info 7.0. Realizaram-se análises de estatísticas descritivas e testes de associação. O teste do Qui-Quadrado foi utilizado para comparação da susceptibilidade antimicrobiana entre homens e mulheres e entre diferentes faixas etárias, adotando-se o Teste Exato de Fisher quando necessário. O odds ratio, com um intervalo de confiança de $95 \%$, foi utilizado na avaliação da razão de chance de isolamento das bactérias. Para comparação da proporção quando cruzadas as variáveis faixa etária e sexo, foi utilizado o teste do Qui-quadrado. Adotou-se um nível de significância de 5\%. Essa pesquisa foi aprovada pelo Comitê de Ética e Pesquisa com Seres Humanos, sob protocolo: 1.053.771. Nenhum conflito de interesse é declarado pelos autores.

\section{Resultados}

Em 2014, foram realizados 4603 exames, sendo registradas 1035 uroculturas positivas (taxa de positividade de $22,48 \%$ ), das quais $89,66 \%$ de pacientes femininas. Como mostrado na Tabela 1 , houve maior prevalência de uroculturas positivas em pacientes com idade acima de 60 anos e as proporções entre os gêneros por faixa etária mostraram-se estatisticamente significativas. Entre 15 e 30 anos, observou-se maior proporção de mulheres. Já o menor valor da relação entre os sexos ocorreu na faixa etária de 1 a 5 anos (desconsiderando-se o grupo de 0 a 1 ano devido à escassez de dados).

Microrganismos Gram-negativos foram os patógenos mais frequentemente isolados, correspondendo a $93,81 \%$ da amostra, a maioria pertencente à família Enterobacteriaceae, com destaque para a Escherichia coli $(77,10 \%)$, principal agente etiológico. Os cocos Gram-positivos corresponderam a 6,19\% dos isolamentos.

Tabela 1. Prevalência de urocultura positiva e média de idade segundo faixa etária e sexo de pacientes atendidos por um laboratório de análises clínicas, em Florianópolis no ano de 2014.

\begin{tabular}{|c|c|c|c|c|c|c|c|c|}
\hline \multirow{2}{*}{ Faixa Etária (anos) } & \multicolumn{2}{|c|}{ Feminino (F) } & \multicolumn{2}{|c|}{ Masculino (M) } & \multicolumn{2}{|c|}{ Total } & \multirow{2}{*}{ Média (anos) } & \multirow{2}{*}{$\mathrm{F} / \mathbf{M}^{\dagger}$} \\
\hline & VA & VR\% & VA & VR\% & VA & VR\% & & \\
\hline $0 \mathrm{a} 1^{*}$ & 0 & 0 & 3 & 2,8 & 3 & 0,29 & 0 & 0 \\
\hline 1 a 5 & 21 & 2,26 & 8 & 7,48 & 29 & 2,80 & 2,17 & 2,62 \\
\hline 5 a 15 & 13 & 1,40 & 2 & 1,87 & 15 & 1,45 & 8,46 & 6,5 \\
\hline 15 a 30 & 134 & 14,44 & 5 & 4,67 & 139 & 13,43 & 23,90 & 26,8 \\
\hline 30 a 60 & 406 & 43,75 & 18 & 16,82 & 424 & 40,97 & 44,51 & 22,55 \\
\hline$\geq 60$ & 354 & 38,15 & 71 & 66,36 & 425 & 41,06 & 75,57 & 4,98 \\
\hline Total & 928 & 100 & 107 & 100 & 1.035 & 100 & & \\
\hline
\end{tabular}

VA: valor absoluto; VR: valor relativo; ${ }^{*}$ Número escassos de dados ${ }^{\dagger} p<0,00001$. 
Conforme demonstrado na Tabela 2, E. coli foi significativamente mais prevalente no sexo feminino, enquanto Proteus mirabilis foi mais prevalente no sexo masculino. Apesar de não ter sido provada significância estatística da diferença de prevalência de Staphylococcus saprophyticus e Klebsiella pneumoniae entre os gêneros, observou-se que as mulheres apresentam 3,17 vezes mais chance de serem acometidas pelo $S$. saprophyticus, enquanto os homens têm 1,38 vezes mais chance de terem a $K$. pneumoniae como agente etiológico da ITU. Nestes, este uropatógeno foi isolado apenas na faixa etária superior a 60 anos, como observado na Tabela 3.

Tabela 2. Frequência por sexo dos uropatógenis isolados de pacientes ambulatoriais em Florianópolis no ano de 2014.

\begin{tabular}{|c|c|c|c|c|}
\hline Feminino $n=928$ & Masculino $\mathrm{n}=107$ & Odds Ratio* & IC $95 \%$ & $\mathbf{p}$ \\
\hline Escherichia coli $(79,20 \%)$ & Escherichia coli $(58,88 \%)$ & $2,6598(\mathrm{M})$ & $1,7538-4,0336$ & $p<0,0001$ \\
\hline Klebsiella pneumoniae $(9,05 \%)$ & Klebsiella pneumoniae $(12,15 \%)$ & $1,3896(H)$ & $0,7461-2,5878$ & $p=0,2997$ \\
\hline Proteus mirabilis $(3,67 \%)$ & Proteus mirabilis $(8,41 \%)$ & $2,4148(H)$ & $1,1251-5,1829$ & $p=0,0237$ \\
\hline Staphylococcus saprophyticus (2,91\%) & Staphylococcus saprophyticus (0,93\%) & $3,1765(\mathrm{M})$ & $0,4273-23,6144$ & $p=0,2588$ \\
\hline Enterococcus faecalis $(2,15 \%)$ & Enterococcus faecalis $(8,41 \%)$ & 4,1694 (H) & $1,8477-9,4086$ & $p=0,0006$ \\
\hline${\text { Outras bactérias gram negativas }{ }^{\dagger},(2,27 \%)}$ & Outras bactérias gram negativas $(11,22 \%)$ & $5,4556(\mathrm{H})$ & $2,6027-11,4357$ & $p<0,0001$ \\
\hline Outras bactérias gram positivas ${ }^{\ddagger},(0,75 \%)$ & Outras bactérias gram positivas, $(0 \%)$ & $1,7499(\mathrm{M})$ & $0,0992-30,8539$ & $p=0,7023$ \\
\hline
\end{tabular}

Tabela 3. Frequência estratificada por sexo e faixa etária em anos dos uropatógenos isolados de pacientes ambulatoriais atendidos por um laboratório de análises clínicas de Florianópolis em 2014.

\begin{tabular}{|c|c|c|c|c|c|c|c|c|c|c|c|c|}
\hline \multirow{2}{*}{ Uropatógenos } & \multicolumn{2}{|c|}{0 a 1} & \multicolumn{2}{|c|}{1 a 5} & \multicolumn{2}{|c|}{5 a 15} & \multicolumn{2}{|c|}{15 a 30} & \multicolumn{2}{|c|}{30 a 60} & \multicolumn{2}{|c|}{60} \\
\hline & F\% & M\% & F\% & M\% & F\% & M\% & F\% & M\% & F\% & M\% & F\% & M\% \\
\hline Escherichia coli & 0 & 33,33 & 71,43 & 12,50 & 69,24 & 50,00 & 76,12 & 60,00 & 81,53 & 77,78 & 78,53 & 60,56 \\
\hline Klebsiella pneumoniae & 0 & 0 & 4,76 & 0 & 7,69 & 0 & 5,23 & 0 & 9,36 & 0 & 10,46 & 18,31 \\
\hline Proteus mirabilis & 0 & 33,33 & 19,05 & 62,50 & 7,69 & 50,00 & 3,73 & 20,00 & 1,97 & 0 & 4,52 & 1,41 \\
\hline Enterococcus faecalis & 0 & 33,33 & 0 & 12,50 & 7,69 & 0 & 0,00 & 0 & 1,97 & 11,11 & 3,11 & 7,04 \\
\hline Staphylococcus saprophyticus & 0 & 0 & 0 & 0 & 7,69 & 0 & 9,70 & 20,00 & 2,95 & 0 & 0,28 & 0 \\
\hline Outras bactérias gram negativas & 0 & 0 & 4,76 & 12,50 & 0 & 0 & 3,73 & 0 & 1,48 & 11,11 & 2,54 & 12,68 \\
\hline Outras bactérias gram positivas & 0 & 0 & 0 & 0 & 0 & 0 & 1,49 & 0 & 0,74 & 0 & 0,56 & 0 \\
\hline Total (\%) & 0 & 100 & 100 & 100 & 100 & 100 & 100 & 100 & 100 & 100 & 100 & 100 \\
\hline
\end{tabular}

F: feminino; M: masculino.

As prevalências de resistência em relação aos antibióticos testados, como resumido no Quadro 1 e demonstrado na Tabela 4, divergem entre os uropatógenos. A E. coli apresentou o perfil mais amplo de resistência, todavia, como apresentado na Tabela 5, esse perfil modifica-se quando estratificamos a resistência antimicrobiana por faixa etária e sexo. Evidenciaram-se diferenças estatisticamente significativas entre os gêneros na faixa etária entre 30-59 anos para o ciprofloxacino, norfloxacino e a gentamicina, com maiores prevalências de resistência em homens, quadro não observado nos pacientes maiores de 60 anos, em que ambos os sexos apresentaram-se resistentes aos dois primeiros antibióticos. Quando comparadas as faixas etárias, os pacientes maiores de 60 anos mostraram maiores resistências às quinolonas do que os mais jovens $(p<0,0001)$. 
Quadro 1. Antimicrobianos com as menores taxas de prevalência de resistência dos uropatógenos mais comuns isolados de pacientes ambulatoriais em Florianópolis.

\begin{tabular}{|cccc|}
\hline E. coli & K. pneumoniae & P. Mirabilis & S. Saprophyticus \\
Gentamicina (7,64\%) & Gentamicina (12,37\%) & Ciprofloxacino (9,68\%) & Nitrofurantoína (0\%) \\
Nitrofurantoína (14,7\%) & Sulfametoxazol + Timetoprima (13,54\%) & Norfloxacino (9,68\%) & Sulfametoxazol + Timetoprima (11,54\%) \\
& Amoxacilina + Clavulanato (17,71\%) & Norfloxacino (3,57\%) \\
& Norfloxacino (19,15\%) & \\
\hline
\end{tabular}

Tabela 4. Taxa de prevalência de resistência antimicrobiana dos principais uropatógenos isolados de pacientes ambulatoriais, em Florianópolis no ano de 2014.

\begin{tabular}{|c|c|c|c|c|c|}
\hline Antibiótico & E. coli* & K. pneumoniae ${ }^{\dagger}$ & P. mirabilis ${ }^{\ddagger}$ & E. faecalis & S. saprophyticus \\
\hline Ampicilina (AMP) & $52,82 \%$ & $100,00 \%$ & $36,59 \%$ & $0,00 \%$ & - \\
\hline Amoxicilina + Clavulanato (AMC) & $33,10 \%$ & $17,71 \%$ & $53,33 \%$ & - & - \\
\hline Cefalotina (CFL) & $56,35 \%$ & $23,16 \%$ & $13,95 \%$ & - & - \\
\hline Cefuroxima (CRX) & $24,89 \%$ & $50 \%$ & $28,57 \%$ & - & - \\
\hline Ácido Nalidíxico (NAL) & $31,58 \%$ & $32,29 \%$ & $11,63 \%$ & - & - \\
\hline Ciprofloxacino (CIP) & $22,73 \%$ & $20,00 \%$ & $9,68 \%$ & $13,79 \%$ & - \\
\hline Norfloxacino (NOR) & $22,21 \%$ & $19,15 \%$ & $9,68 \%$ & - & $3,57 \%$ \\
\hline Gentamicina (GEN) & $7,64 \%$ & $12,37 \%$ & $0,00 \%$ & - & - \\
\hline
\end{tabular}

Tabela 5. Resistência antimicrobiana das capas de Escherichia coli isoladas em Florianópolis no ano de 2014 estratificadas por sexo e faixa etária.

\begin{tabular}{|c|c|c|c|c|c|c|c|c|c|c|}
\hline \multirow{2}{*}{ Antibiótico } & \multicolumn{5}{|c|}{ Feminino (anos) } & \multicolumn{5}{|c|}{ Masculino (anos) } \\
\hline & $0-14^{*}$ & $15-29 *$ & $30-59$ & $60-79$ & 80 & $0-14^{*}$ & $15-29 *$ & $30-59$ & $60-79$ & 80 \\
\hline Ampicilina & $59,26 \%$ & $40,95 \%$ & $46,67 \%$ & $59,54 \%$ & $63,81 \%$ & - & - & $64,29 \%$ & $62,96 \%$ & $75 \%$ \\
\hline Amoxicilina + Clavulanato & $31,25 \%$ & $26,53 \%$ & $30,13 \%$ & $35,30 \%$ & $35,82 \%$ & - & - & - & $44,44 \%$ & $58,33 \%$ \\
\hline Sulfametoxazol + Trimetoprima & $18,52 \%$ & $25 \%$ & $27,79 \%$ & $38,15 \%$ & $39,05 \%$ & - & - & $42,86 \%$ & $29,63 \%$ & $56,25 \%$ \\
\hline Cefalotina & $51,85 \%$ & $53,84 \%$ & $50,76 \%$ & $62,79 \%$ & $60,58 \%$ & - & - & $57,14 \%$ & $70,37 \%$ & $75 \%$ \\
\hline Cefuroxima & $25 \%$ & $14,51 \%$ & $16 \%$ & $28,44 \%$ & $43,75 \%$ & - & - & - & $31,58 \%$ & $50 \%$ \\
\hline Nitrofurantoína & $18,52 \%$ & $4,76 \%$ & $10,91 \%$ & $15,60 \%$ & $24,04 \%$ & - & - & $28,57 \%$ & $22,22 \%$ & $25 \%$ \\
\hline Ácido Nalidíxico & $3,70 \%$ & $14,28 \%$ & $21,15 \%$ & $43,35 \%$ & $57,14 \%$ & - & - & $50 \%$ & $51,85 \%$ & $62,50 \%$ \\
\hline Ciprofloxacino & - & $6,66 \%$ & $12,73^{\dagger}$ & $29,48 \%$ & $45,71 \%$ & - & - & $46,15 \%^{\dagger}$ & $40,74 \%$ & $62,50 \%$ \\
\hline Norfloxacino & - & $6,80 \%$ & $12,38 \%^{\dagger}$ & $28,90 \%$ & $43,81 \%$ & - & - & $42,86 \%^{\dagger}$ & $40,74 \%$ & $62,50 \%$ \\
\hline Gentamicina & $0 \%$ & $4,75 \%$ & $6,34 \%^{\dagger}$ & $6,94 \%$ & $10,48 \%^{\dagger}$ & - & - & $28,57 \%^{\dagger}$ & $11,11 \%$ & $31,25 \%^{\dagger}$ \\
\hline
\end{tabular}

${ }^{*}$ Devido ao número escasso de dados masculinos nas amostras entre 0-14 e 15-29 anos, calculou-se a resistência geral nessas faixas etárias; ${ }^{\dagger} p<5 \%$ quando a resistência antimicrobiana por sexo na mesma faixa etária. 


\section{Discussão}

Resumo dos principais achados do estudo

Os resultados sugerem que a idade e o sexo são variáveis determinantes na frequência de isolamento dos uropatógenos e na prevalência da susceptibilidade antimicrobiana e demonstram que os microrganismos mais prevalentes já não respondem satisfatoriamente a parte dos antimicrobianos amplamente utilizados na região. Além disso, evidenciou-se que, mesmo no contexto da atenção primária, deve-se considerar o potencial envolvimento de bactérias resistentes a múltiplos antibióticos, sobretudo nas infecções urinárias dos homens e dos idosos.

Fortalezas e limitações do estudo

Como são escassos os trabalhos de vigilância epidemiológica e laboratorial em Florianópolis, o presente estudo tem elevada importância, embora apresente algumas limitações: variáveis relevantes como manifestações clínicas, gravidade do quadro, comorbidades associadas, história de infecção prévia e exposição recente a antibióticos não foram analisadas. Além disso, como a maior parte das ITUs não complicadas são tratadas empiricamente, as amostras de urina enviadas para a cultura tendem a ser de pacientes com quadro clínico complicado, infecções recorrentes ou com falha à terapia inicial, o que gera um viés de seleção. Sendo assim, os resultados apresentados podem superestimar a resistência antimicrobiana local das ITUs comunitárias.

Comparação com a literatura já existente

A ITU pode ser classificada de acordo com a sua localização no trato urinário em infeç̧ão baixa (cistite, uretrite) ou alta (pielonefrite, abscesso renal) e de acordo com a sua gravidade em complicada ou não complicada. ${ }^{1} \mathrm{O}$ quadro é considerado complicado na existência de fatores que predispõem à persistência ou recidiva da infecção como: presença de corpos estranhos (cálculos, cateter vesical de demora ou outros dispositivos de drenagem), obstrução, imunossupressão e alterações funcionais como refluxo vesicoureteral e retenção urinária da doença neurológica. ${ }^{1,19,20}$

A finalidade dessa classificação reside no fato de que há maior dificuldade no manejo e desfechos mais graves nas infecções complicadas. ${ }^{20}$ Sendo assim, o exame complementar pode ser dispensado nas mulheres adultas com alta probabilidade de cistite não complicada, ${ }^{6}$ ou seja, naquelas com combinação de disúria e polaciúria na ausência de corrimento vaginal, quadro que eleva para $96 \%$ a probabilidade diagnóstica. ${ }^{21}$ Já para o restante dos casos (mulheres com cistite complicada ou pielonefrite e ITU em homens ou crianças), uma urocultura com antibiograma deve ser solicitada para garantir que a escolha antimicrobiana empírica foi apropriada ou orientar a modificação terapêutica. ${ }^{6}$ 
A principal etiologia da ITU é a ascensão uretral de enterobactérias ${ }^{6} \mathrm{e}$, assim como encontrado em outras casuísticas, ${ }^{10,22,23}$ E. coli, K. pneumoniae e $P$. mirabilis foram os uropatógenos mais prevalentes em ambos os sexos. $A E$. colié o microrganismo mais frequentemente isolado, tanto em pacientes hospitalizados como nos ambulatoriais. ${ }^{23}$ As ITUs nos lactentes, contudo, têm a peculiaridade de apresentar uma frequência de uropatógenos variável. ${ }^{24}$ Já entre os pacientes masculinos, sobretudo nas crianças, o P. mirabilis é um importante agente causal. ${ }^{24,25}$ Além desse microrganismo ter sido mais prevalente entre os homens, os meninos entre 0 a 15 anos representaram o único grupo analisado em que a $E$. coli não foi a bactéria mais isolada, tendo o $P$. mirabilis sido o principal responsável pelas infecções daqueles entre 1 a 5 anos. Esse conhecimento é de fundamental importância para a prescrição tanto dos lactentes, em que se deve considerar esquemas antimicrobianos empíricos de amplo espectro, como dos meninos, uma vez que a sensibilidade antimicrobiana do $P$. mirabilis não coincide com a da E.coli. ${ }^{24}$

Com exceção do primeiro ano de vida, essa infecção - devido a condições anatômicas propícias: uretra mais curta, maior colonização periuretral e ausência de substâncias antibacterianas do líquido prostático - é mais frequente entre as mulheres. ${ }^{6}$ Apesar de ocorrer em todas as idades, ${ }^{22}$ a ITU é mais prevalente nas jovens com vida sexual ativa e nos adultos com mais de 60 anos, ${ }^{23}$ havendo neste último grupo maior ocorrência de infecções complicadas, uma vez que nos idosos há o predomínio de doenças neuromusculares, demência, diabetes e maior frequência de cálculos, tumores e instrumentação. ${ }^{20}$

A maior parte das mulheres deste estudo encontrava-se na faixa entre 15 e 60 anos e, entre as mais jovens, o $S$. saprophyticus - patógeno sabidamente relacionado à atividade sexual ${ }^{24}$ - foi o segundo agente etiológico mais comum. As idosas representaram a segunda faixa etária mais acometida no sexo feminino, destacando-se o prolapso genitourinário como uma condição comum e que, por resultar no esvaziamento incompleto da bexiga, ${ }^{20}$ pode explicar, juntamente com a menopausa, ${ }^{1}$ a elevada incidência da doença nesse grupo. Já em relação aos pacientes masculinos, as maiores prevalências de prostatite e de obstrução uretral decorrente de hipertrofia prostática após os 50 anos justificam que a maioria dos homens pertencentes à amostra tivesse mais de 60 anos. ${ }^{6}$

Por ser a responsável pela maior parte dos casos de ITU, não se deve utilizar no tratamento empírico antibióticos para os quais haja uma resistência local da E. coli maior que $20 \% .^{6,8}$ O perfil de resistência das amostras desse uropatógeno revelou que, na comunidade estudada, a gentamicina e a nitrofurantoína apresentaram as menores prevalências de resistência, o que vai ao encontro dos dados existentes na literatura ${ }^{22,23,26}$ e configura a nitrofurantoína, antimicrobiano para o qual já foi provada a ausência de resistência cruzada ${ }^{27}$ e a atividade contra cepas de E. coli resistentes a múltiplas drogas, ${ }^{28}$ como a opção ideal no tratamento das cistites não complicadas. A resistência ao sulfametoxazol/trimetoprima foi semelhante a diversos estudos brasileiros ${ }^{7,22,23,26}$ e provavelmente reflete o uso generalizado e indevido deste antimicrobiano de baixo custo para o tratamento de ITU no País. ${ }^{10}$ Já a ampicilina, a cefalotina e a amoxacilina+clavulanato apresentaram susceptibilidade $<80 \%$ em todas as faixas etárias analisadas, indicando que esses antibióticos devem somente ser utilizados após liberação do resultado do antibiograma.

A sensibilidade da $E$. coli menor que $77,79 \%$ às fluoroquinolonas contrasta com uma susceptibilidade maior que $89 \%$ em Florianópolis na década de $90,{ }^{29}$ e esse aumento na resistência também foi observado por outros estudos nacionais, principalmente a partir dos anos 2000 , quando houve incremento na prescrição desses antibióticos. ${ }^{13,14}$ Contrariando as recomendações atuais, como identificado em algumas unidades 
básicas de saúde, onde o norfloxacino foi o segundo antimicrobiano mais prescrito para ITU, ${ }^{5}$ percebe-se uma tendência de preferência dos médicos para agentes de amplo espectro. ${ }^{30}$

Aexposição prévia a fluoroquinolonas é um fator de risco independente para a aquisição de resistência ${ }^{14}$ e as consequências ambientais decorrentes do uso difundido desse antibiótico, caracteristicamente excretado na forma não metabolizada na urina e assim presente em fontes de água, esgoto e alimentos, ${ }^{10,31}$ bem como a associação entre a resistência da $E$. coli às fluoroquinolonas com maiores resistências a outros antimicrobianos $^{32}$ reforçam a gravidade do quadro e a necessidade urgente de uma política de redução no consumo dessas drogas. ${ }^{26}$

As maiores taxas de resistência às fluoroquinolonas entre os homens em relação às mulheres e em pacientes de ambos os sexos com mais de 60 anos em relação às demais faixas etárias também foram observadas por estudos nacionais ${ }^{13}$ e internacionais ${ }^{11}$ e podem ser explicadas pelo fato de que as infecções complicadas apresentam maior tendência à falha terapêutica, o que leva a uma exposição cumulativa aos antimicrobianos e consequente seleção de microrganismos resistentes. ${ }^{13,20}$ Entre os homens, mesmo nos mais jovens, a ITU é considerada complicada, devendo-se suspeitar de contaminação por germes resistentes naqueles com história de ITU prévia e instrumentação. ${ }^{20}$ Já os idosos, além da maior frequência de cateterização urinária, apresentam maior susceptibilidade a internações hospitalares, ambos considerados fatores de risco independentes para a resistência às fluoroquinolonas. ${ }^{10}$

Os esquemas terapêuticos em Pediatria para ITUs usualmente incluem cefalosporinas, amoxicilina+clavulanato ou sufametoxazol/trimetoprima. ${ }^{33} \mathrm{Em}$ Florianópolis, porém, encontrou-se sensibilidade diminuída à amoxacilina+clavulanato, à cefalotina e à cefuroxima nos pacientes entre 0 e 14 anos, o que contrasta com os achados de São Paulo. ${ }^{24}$ Assim como outros estudos haviam sugerido que adultos e crianças apresentam diferenças nos padrões de resistência à $E$. coli, ${ }^{3}$ na comunidade estudada o sulfametoxazol/trimetoprima, ao contrário das demais faixas etárias, mostrou-se uma boa opção na terapia empírica de pacientes pediátricos. Apesar de apresentar sensibilidade de 81,48\%, o uso da nitrofurantoína está contraindicado no tratamento de crianças febris, uma vez que essa droga não atinge concentrações teciduais e séricas terapêuticas para pielonefrite e urosepsis. ${ }^{33}$

De acordo com as recomendações internacionais mais atuais, nitrofurantoína, sulfametoxazol/ trimetoprima e fosfomicina são os agentes de primeira linha para o tratamento empírico de ITU não complicada em mulheres. ${ }^{8} \mathrm{Na}$ população estudada, a nitrofurantoína consiste na melhor escolha terapêutica, já o sulfametoxazol/trimetoprim não deve ser usado empiricamente. Apesar da resistência ainda intermediária a baixa da $E$. coli às quinolonas em mulheres menores de 60 anos, esses antibióticos devem ser resguardados para o tratamento de ITUs mais graves, como a cistite complicada e a pielonefrite. ${ }^{6}$

Guidelines recomendam que o ciprofloxacino seja o agente escolhido no tratamento da pielonefrite não complicada e, nas regiões onde a resistência a esse antibiótico é maior que $10 \%$, orienta-se uma dose inicial de ceftriaxona ou 24 horas de um aminoglicosídeo antes de iniciar o tratamento com a quinolona8. Tal cuidado deveria ser tomado nas mulheres entre 30-59 anos, as quais apresentaram resistência de $12,73 \%$ ao antimicrobiano de escolha.

A Gentamicina mostrou-se a única opção entre as idosas maiores de 80 anos e, em relação aos homens, além de as resistências terem sido elevadas em todas as faixas etárias, naqueles entre 30-59 anos e nos maiores de 80 anos nenhum antibiótico analisado apresentou resistência menor que $20 \%$. Assim como 
demonstrado por outros estudos, ${ }^{11,13}$ o tratamento empírico dos pacientes com ITU complicada consiste em um desafio terapêutico que não apresenta soluções evidentes, sendo algumas alternativas descritas: tratamento com cefalosporinas de terceira e quarta gerações, administração de dose inicial de um agente parenteral de largo espectro (carbapenens ou piperacilina+tazobactam) ou a utilização da fosfomicina. ${ }^{13}$

Esse último antimicrobiano merece destaque, uma vez que se tem provado que a fosfomicina, devido a sua estrutura química e mecanismo de ação, é poupada de vários mecanismos de resistência, incluindo aqueles contra as fluoroquinolonas e os associados à produção de beta lactamase de espectro estendido, o que suporta a ideia de que essa droga é uma valiosa opção no tratamento da cistite complicada, resultando em melhores desfechos do que as drogas habitualmente usadas para as bactérias multirresistentes. ${ }^{34}$ Todavia, assim como ocorreu nessa pesquisa, esse agente não é comumente testado nos antibiogramas, ${ }^{28}$ o que dificulta a sua maior utilização, visto que os médicos tendem a prescrever uma droga apenas se a sua sensibilidade for reportada no resultado do antibiograma. ${ }^{4}$

Implicações para a pesquisa na área e/ou para a prática dos profissionais

É fundamental que o médico utilize os conhecimentos acerca da relação entre sexo e idade com a incidência de ITU, com a frequência de isolamento dos uropatógenos e a prevalência da susceptibilidade antimicrobiana a fim de decidir com que probabilidade considerar o diagnóstico e de utilizar regimes de tratamento adaptados ao perfil demográfico do paciente, evitando, assim, gastos desnecessários por falha terapêutica. Além disso, a gravidade do quadro clínico e a existência de fatores de risco para infecção complicadas devem ser valorizadas na escolha antimicrobiana devido ao potencial envolvimento de bactérias sensíveis apenas aos antibióticos de largo espectro. Sugerem-se futuras pesquisas sobre a avaliação das variáveis não analisadas pela presente casuística, com a inclusão de outros antimicrobianos como a fosfomicina.

\section{Conclusão}

As modificações do perfil de susceptibilidade antimicrobiana nas últimas décadas em Florianópolis e as divergências em relação aos dados encontrados em outros países e estados da federação reforçam a ideia de aumento da resistência com o tempo, associado principalmente ao uso indiscriminado de determinadas drogas e às pressões seletivas locais, demonstrando que o manejo baseado apenas em diretrizes internacionais encontra-se completamente inadequado.

O cenário evidenciado indica a necessidade urgente de implementação de programas locais de gestão de antimicrobianos que prezem o uso racional, em especial dos antibióticos de largo espectro como as quinolonas, diminuindo a pressão seletiva sobre os mesmos, e a elaboração de uma rede sentinela em que se mantenham atualizados os dados referentes aos padrões de resistência antimicrobiana locais, permitindo que a decisão terapêutica, baseada em evidências, seja mais efetiva.

\section{Referências}

1. Duncan BB, Schmidt MI, Giugliane ERJ, Duncan MS, Giugliane C. Medicina ambulatorial: condutas de atenção primária baseadas em evidências. 4. ed. Porto Alegre: Artmed; 2013.1976 p.

2. Kumar S, Dave A, Wolf B, Lerma EV. Urinary tract infections. Dis Mon. 2015;61(2):45-59. DOI: http://dx.doi.org/10.1016/j. disamonth.2014.12.002 
3. Gusso GFD. Diagnóstico de demanda em Florianópolis utilizando a Classificação Internacional de Atenção Primária: $2^{\text {a }}$ edição (CIAP-2) [tese]. São Paulo. Universidade de São Paulo; 2009. 168 p. [Citado 10 Set 2014]. Disponível em: http://www.teses.usp.br/teses/disponiveis/5/5159/tde-08032010-164025/pt-br.php

4. Coupart C, Pradier C, Degand N, Hofliger P, Pulcini C. Selective reporting of antibiotic susceptibility data improves the appropriateness of intended antibiotic prescriptions in urinary tract infections: a case-vignette randomised study. Eur J Clin Microbiol Infect Dis. 2013;32(5):627-36. DOI: http://dx.doi.org/0.1007/s10096-012-1786-4

5. Tavares NUL, Bertoldi AD, Muccillo-Baisch AL. Prescrição de antimicrobianos em unidades de saúde da família no Sul do Brasil. Cad. Saúde Pública. 2008;24(8):1791-800. DOI: http://dx.doi.org/10.1590/S0102-311X2008000800008

6. Gusso G, Lopes JMC. Tratado de medicina de família e comunidade: princípios, formação e prática. Porto Alegre: Artmed; 2012. $1354 \mathrm{p}$.

7. Teichmann A, Agra HN, Nunes L de S, da Rocha MP, Renner JD, Possuelo LG, et al. Antibiotic resistance and detection of the sul2 gene in urinary isolates of Escherichia coli in patients from Brazil. J Infect Dev Ctries. 2014;8(1):39-43. DOI: http://dx.doi.org/10.3855/jidc.3380

8. Gupta K, Hooton TM, Naber KG, Wullt B, Colgan R, Miller LG, et al.; Infectious Diseases Society of America; European Society for Microbiology and Infectious Diseases. International clinical practice guidelines for the treatment of acute uncomplicated cystitis and pyelonephritis in women: A 2010 update by the Infectious Diseases Society of America and the European Society for Microbiology and Infectious Diseases. Clin Infect Dis. 2011;52(5):e103-20. DOI: http://dx.doi. org/10.1093/cid/ciq257

9. Cuba GT, Pignatari AC, Patekoski KS, Luchesi LJ, Kiffer CR. Pharmacodynamic profiling of commonly prescribed antimicrobial drugs against Escherichia coli isolates from urinary tract. Braz J Infect Dis. 2014;18(5):512-7. DOI: http:// dx.doi.org/10.1016/j.bjid.2014.01.008

10. Salles MJ, Zurita J, Mejía C, Villegas MV; Latin America Working Group on Bacterial Resistance. Resistant Gram-negative infections in the outpatient setting in Latin America. Epidemiol Infect. 2013;141(12):2459-72. DOI: http://dx.doi.org/10.1017/ S095026881300191X.

11. Swami SK, Liesinger JT, Shah N, Baddour LM, Banerjee R. Incidence of antibiotic-resistant Escherichia coli bacteriuria according to age and location of onset: a population-based study from Olmsted County, Minnesota. Mayo Clin Proc. 2012;87(8):753-9. DOI: http://dx.doi.org/10.1016/j.mayocp.2012.02.025

12. Schmiemann G, Gágyor I, Hummers-Pradier E, Bleidorn J. Resistance profiles of urinary tract infections in general practice - an observational study. BMC Urol. 2012;12:33. DOI: http://dx.doi.org/10.1186/1471-2490-12-33

13. Rocha JL, Tuon FF, Johnson JR. Sex, drugs, bugs, and age: rational selection of empirical therapy for outpatient urinary tract infection in an era of extensive antimicrobial resistance. Braz J Infect Dis. 2012;16(2):115-21. DOI: http://dx.doi. org/10.1590/S1413-86702012000200002

14. Andrade SS, Sader HS, Jones RN, Pereira AS, Pignatari AC, Gales AC. Increased resistance to first-line agents among bacterial pathogens isolated from urinary tract infections in Latin America: time for local guidelines? Mem Inst Oswaldo Cruz. 2006;101(7):741-8. DOI: http://dx.doi.org/10.1590/S0074-02762006000700006

15. American Society for Microbiology. Manual of antimicrobial susceptibility testing. Seattle: American Society for Microbiology; 2005. [Online]. [Citado 18 Jul 2016]. Disponível em: http://www.asm.org/index.php/34-international/asm-meetings-andconferences/274-antimicrobial-susceptibility-testing-manual

16. Clinical and Laboratory Standards Institute. Methods for Dilution Antimicrobial Susceptibility Tests for Bacteria That Grow Aerobically; Approved Standard-Ninth Edition. CLSI document M07-A9; ISBN 1-56238-784-7. Wayne: Clinical and Laboratory Standards Institute; 2012.

17. Brasil. ANVISA. Nota Técnica No 01/2013: Medidas de prevenção e controle de infecções por enterobactérias multirresistentes. Agência Nacional de Vigilância Sanitária. [Online] 17 de Abr. de 2013. [Citado 18 Jul 2014]. Disponível em: http://portal.anvisa.gov.br/documents/33852/271858/Nota+t\%C3\%A9cnica+n\%C2\%BA+01+de+2013/5be898537eca-4b4b-98e4-5096b9f5a2ec

18. Silveira ACO, Souza HAPHM, Furtado FD, Albini BP, Albini CA. Quando e como valorizar culturas de urina polimicrobianas no laboratório de microbiologia clínica. J Bras Patol Med Lab. 2010;46(4):289-94. DOI: http://dx.doi.org/10.1590/S167624442010000400005 
19. Levison ME, Kaye D. Treatment of complicated urinary tract infections with an emphasis on drug-resistant gram-negative uropathogens. Curr Infect Dis Rep. 2013;15(2):109-15. DOI: http://dx.doi.org/10.1007/s11908-013-0315-7.

20. Kaye D. Complicated Urinary Tract Infection in the Geriatric Population. Curr Geriatr Rep. 2015;4(1):79-86. DOI: http://dx.doi. org/10.1007/s13670-014-0109-2.

21. Bent S, Nallamothu BK, SimeI DL, Fihn SD, Saint S. Does this woman have an acute uncomplicated urinary tract infection? JAMA. 2002;287(20):2701-10. DOI: http://dx.doi.org/10.1001/jama.287.20.2701

22. Braoios A, Turatti TF, Meredija LCS, Campos TRS, Denadai FHM. Infecções do trato urinário em pacientes não hospitalizados: etiologia e padrão de resistência aos antimicrobianos. J Bras Patol Med Lab. 2009;45(6):449-56. DOI: http://dx.doi.org/10.1590/S1676-24442009000600003

23. Santana TCFS, Pereira EMM, Monteiro SG, Carmo MS, Turri RJG, Figueiredo PMS. Prevalência e resistência bacteriana aos agentes antimicrobianos de primeira escolha nas infecções do trato urinário no município de São Luís-MA. Rev Patol Trop. 2012;41(4):409-18.

24. Lo DS, Shieh HH, Ragazzi SLB, Koch VHK, Martinez MB, Gilio AE. Infecção urinária comunitária: etiologia segundo idade e sexo. J Bras Nefrol. 2013;35(2):93-8. DOI: http://dx.doi.org/10.5935/0101-2800.20130016

25. Koch VH, Zuccolotto SM. Infecção do trato urinário: em busca das evidências. J Pediatr (Rio J.). 2003;79(Supl 1):97-106. DOI: http://dx.doi.org/10.1590/S0021-75572003000700011

26. Miranda EJ, Oliveira GS, Roque FL, Santos SR, Olmos RD, Lotufo PA. Susceptibility to antibiotics in urinary tract infections in a secondary care setting from 2005-2006 and 2010-2011, in São Paulo, Brazil: data from 11,943 urine cultures. Rev Inst Med Trop São Paulo. 2014;56(4):313-24. DOI: http://dx.doi.org/10.1590/S0036-46652014000400009

27. Katzung BG. Farmacologia: básica e clínica. 10. ed. Porto Alegre: AMGH; 2010. 1046 p.

28. Sanchez GV, Baird AM, Karlowsky JA, Master RN, Bordon JM. Nitrofurantoin retains antimicrobial activity against multidrugresistant urinary Escherichia coli from US outpatients. J Antimicrob Chemother. 2014;69(12):3259-62. DOI: http://dx.doi. org/10.1093/jac/dku282

29. Silva GV. Sensibilidade dos Uropatógenos de Origem Comunitária e Hospitalar aos Antimicrobianos [monografia]. Florianópolis: Universidade Federal de Santa Catarina; 1998. [Citado 1 Abr 2015]. Disponível em: https://repositorio.ufsc. $\mathrm{br} / \mathrm{bitstream} / \mathrm{handle} / 123456789 / 113602 / 253526$. pdf? sequence $=1$

30. Shapiro DJ, Hicks LA, Pavia AT, Hersh AL. Antibiotic prescribing for adults in ambulatory care in the USA, 2007-09. J Antimicrob Chemother. 2014;69(1):234-40. DOI: http://dx.doi.org/10.1093/jac/dkt301

31. Kiffer CR, Camargo EC, Shimakura SE, Ribeiro PJ Jr, Bailey TC, Pignatari AC, et al. A spatial approach for the epidemiology of antibiotic use and resistance in community-based studies: the emergence of urban clusters of Escherichia coli quinolone resistance in Sao Paulo, Brasil. Int J Health Geogr. 2011;10:17. DOI: http://dx.doi.org/10.1186/1476-072X-10-17

32. Karlowsky JA, Hoban DJ, DeCorby MR, Laing NM, Zhanel GG. Fluoroquinolone-resistant urinary isolates of escherichia coli from outpatients are frequently multidrug resistant: results from the North American Urinary Tract Infection Collaborative Alliance-Quinolone Resistance Study. Antimicrob Agents Chemother. 2006;50(6):2251-4. DOI: http://dx.doi.org/10.1128/ AAC.00123-06

33. Subcommittee on Urinary Tract Infection, Steering Committee on Quality Improvement and Management, Roberts KB. Urinary tract infection: clinical practice guideline for the diagnosis and management of the initial UTI in febrile infants and children 2 to 24 months. Pediatrics. 2011;128(3):595-610. DOI: http://dx.doi.org/10.1542/peds.2011-1330

34. Falagas ME, Kastoris AC, Kapaskelis AM, Karageorgopoulos DE. Fosfomycin for the treatment of multidrug-resistant, including extended-spectrum beta-lactamase producing, Enterobacteriaceae infections: a systematic review. Lancet Infect Dis. 2010;10(1):43-50. DOI: http://dx.doi.org/10.1016/S1473-3099(09)70325-1 